\title{
An open-source portable device for the determination of fluoride in drinking water.
}

\author{
EUGENIO H. OTAL*1,4, MANUELA LETICIA KIM ${ }^{1,4}$, STEFFEN DIETRICH ${ }^{5}$, RYOGO \\ TAKADA $^{6}$, SHINJI NAKAYA ${ }^{6}$, MUTSUMI KIMURA*1,2,3 \\ ${ }^{1}$ Department of Chemistry and Materials, Faculty of Textile Science and Technology, Shinshu University, \\ Japan \\ ${ }^{2}$ COI Aqua-Innovation Center, Shinshu University, Japan \\ ${ }^{3}$ Research Initiative for Supra-Materials, Shinshu University, Japan \\ 4 Unidad de Investigación y Desarrollo de las Ingenierías (UIDI), CONICET, FRBA-UTN, Medrano 951 \\ (C1179AAQ), Buenos Aires, Argentina. \\ ${ }^{5}$ Fritz \& Dietrich GbR, FRIDIE Interactive, Immenhofer Straße. 17, 70180 Stuttgart, Germany \\ ${ }^{6}$ Department of Water Environment and Civil Engineering, Shinshu University, Nagano, Japan
}

\section{Table of Contents}

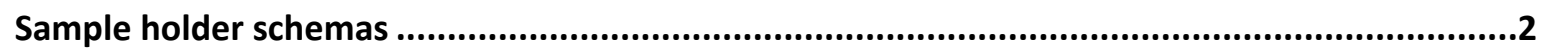

Complexes stability constants......................................................................................4

Visible spectra ......................................................................................................5

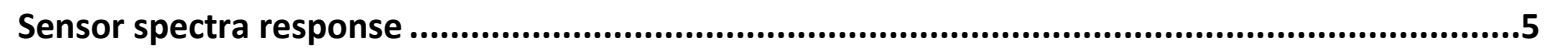

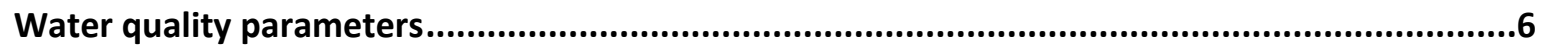

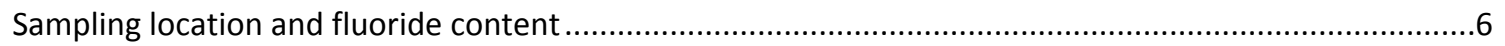

Arduino circuit ...................................................................................................7

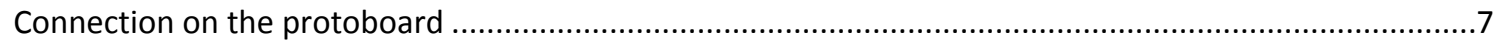

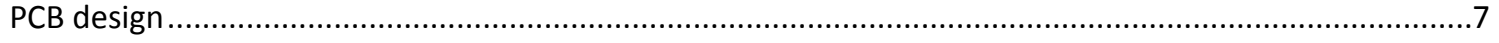

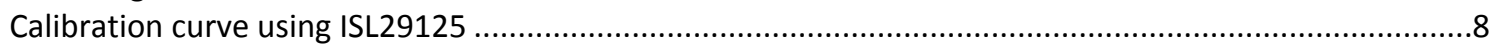

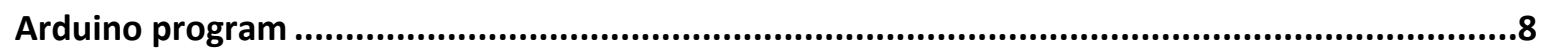

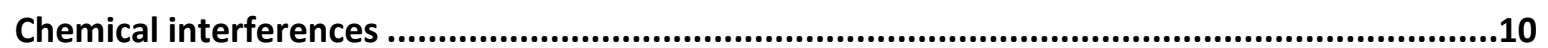

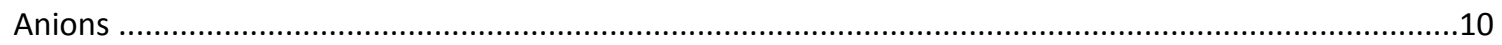

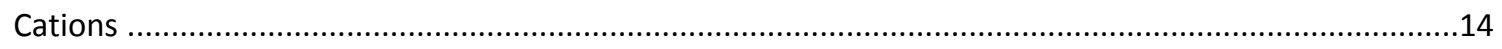

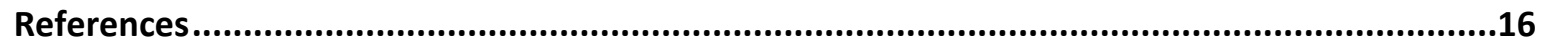


Sample holder schemas
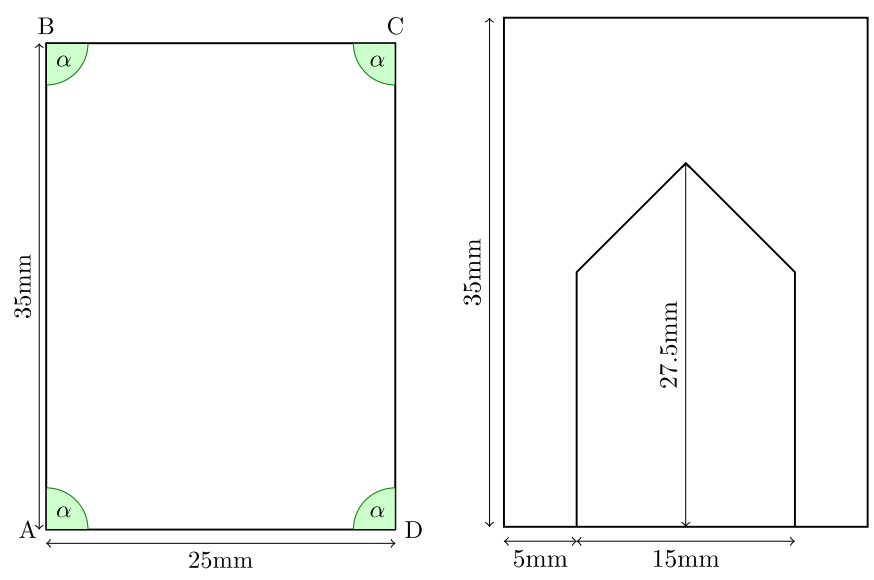

Fig S1 - Sample holder schema used for color intensity quantification in test strips defined in figure 1. Left) back side of the sample holder, right) front side of the sample holder, and cotton substrate dimensions. $\alpha=90^{\circ}$.

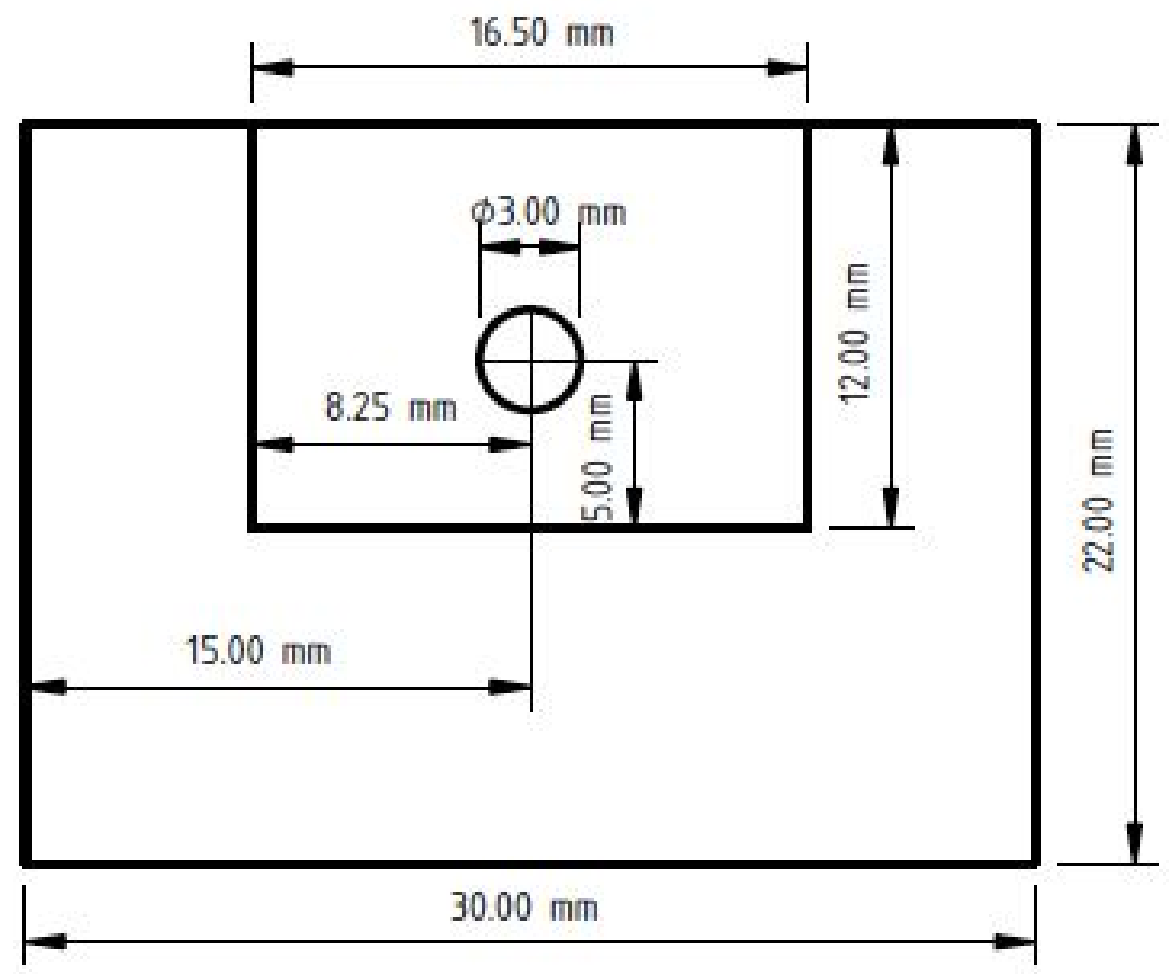



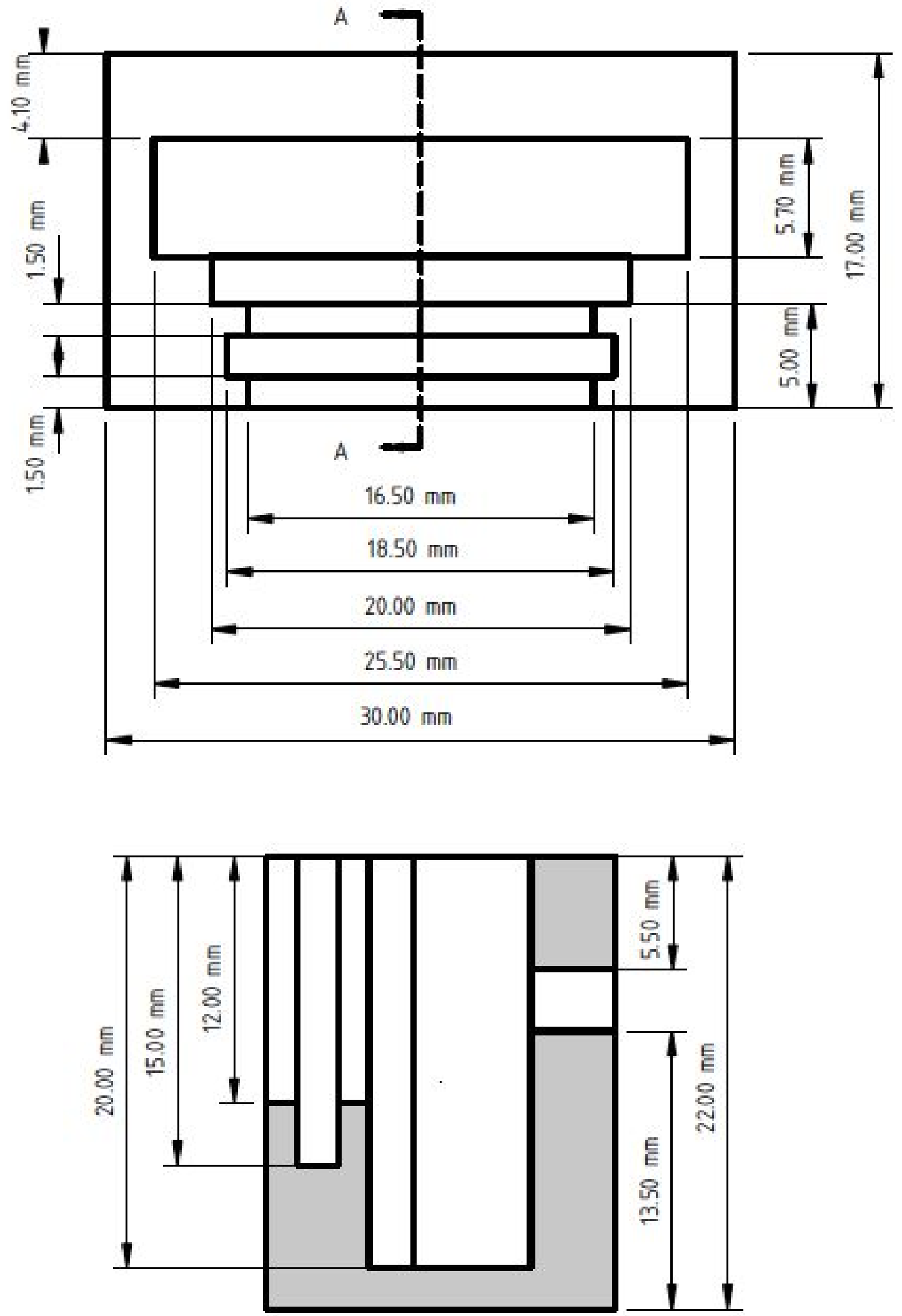


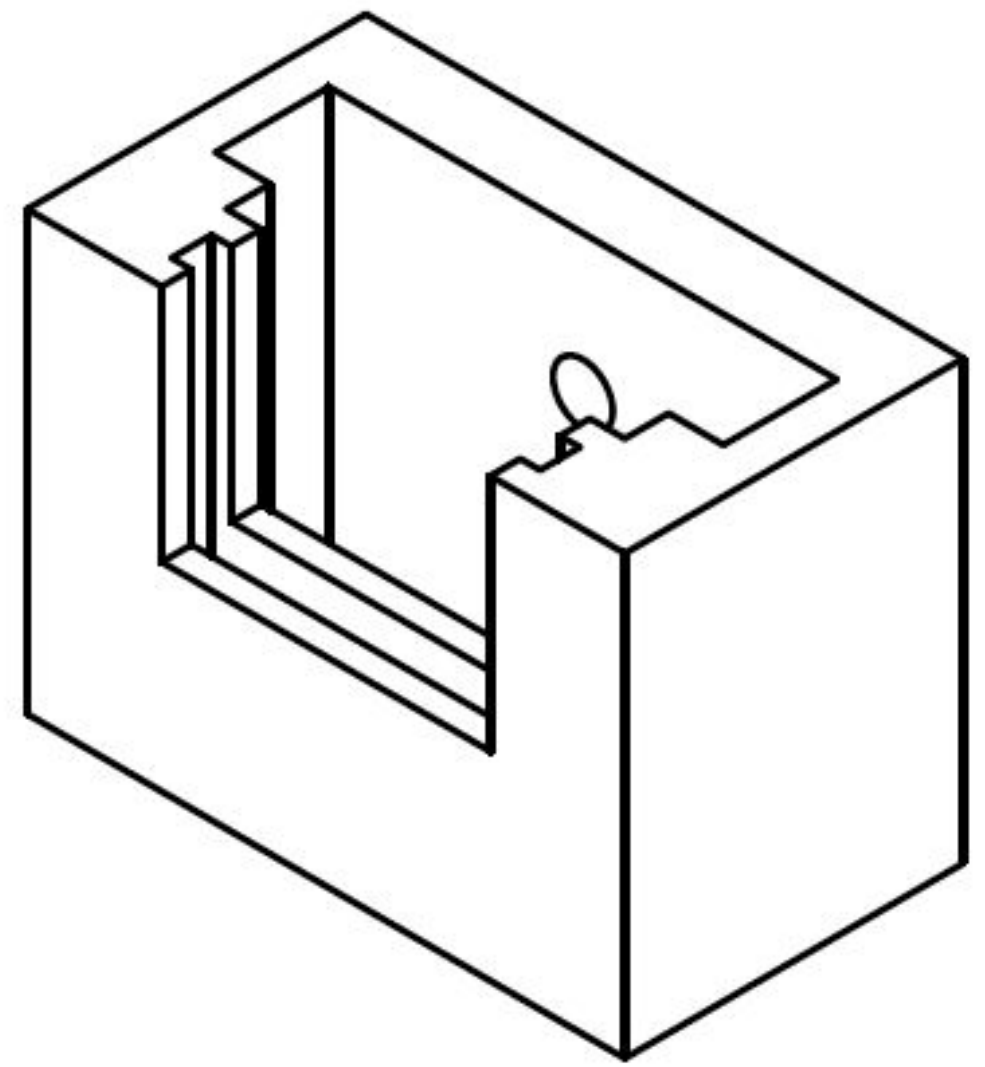

Fig S2 - Technical -drawing, front, top, cut along A-A and perspective view, of the of the LED and ISL29125 holder.

Complexes stability constants

\begin{tabular}{|l|l|l|}
\hline & Fe-SCN $^{1}$ & Fe-F $^{2}$ \\
\hline $\log \beta 1$ & 2.09 & 5.5 \\
\hline $\log \beta 2$ & 3.30 & 9.7 \\
\hline $\log \beta 3$ & & 12.7 \\
\hline $\log \beta 4$ & & 14.9 \\
\hline $\log \beta 5$ & & 15.4 \\
\hline
\end{tabular}

Table S1 -Stability constants of Fe/SCN and Fe/F complex. 
Visible spectra

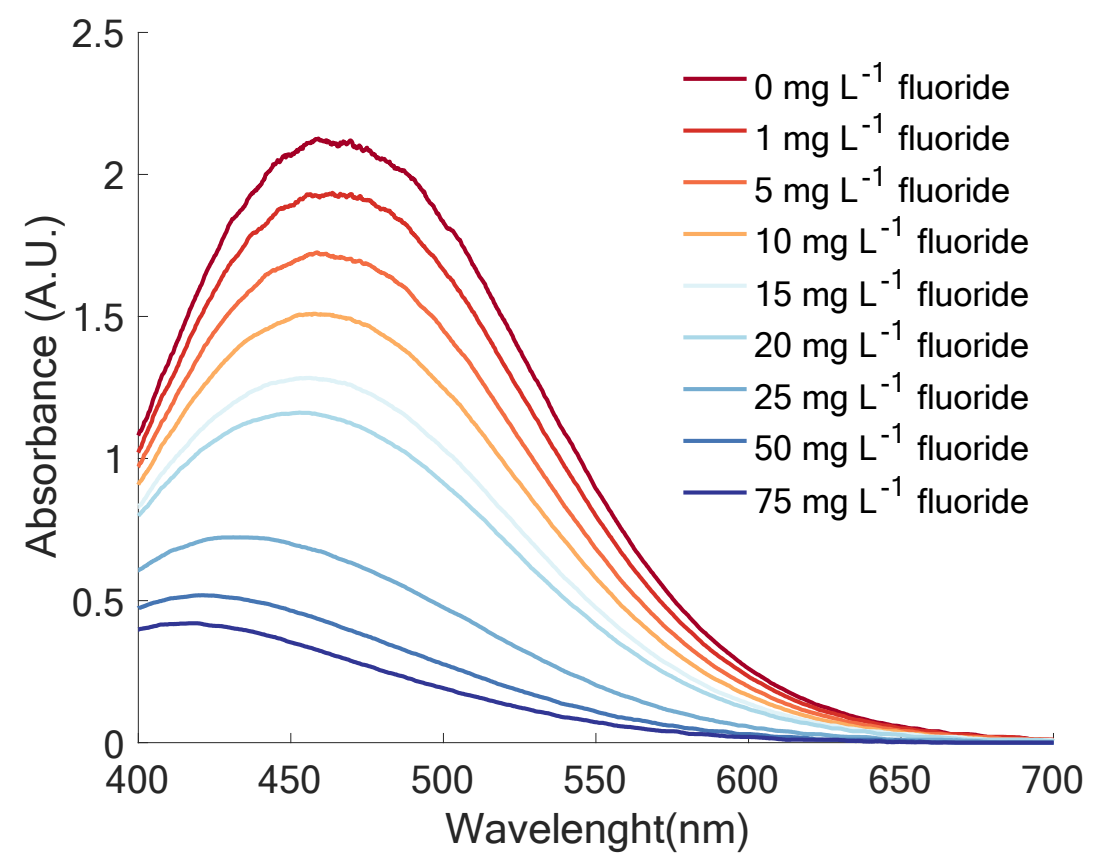

Fig S3 - Visible absorption spectra of Fe-SCN system with different concentrations of fluorides.

Sensor spectra response

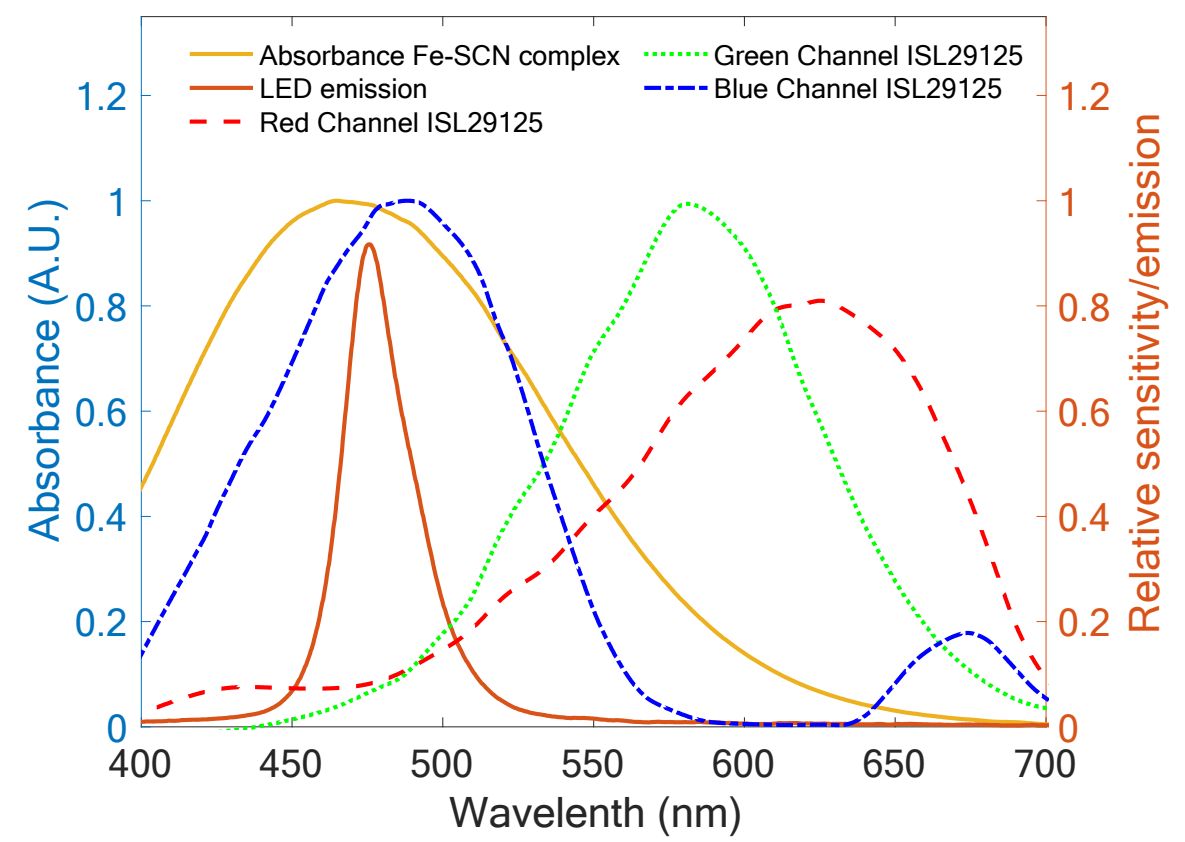

Fig S4 - Normalized spectral response for ambient light sensing for ISL29125 sensor, LED emission and Fe-SCN complex absorbance in the visible region. 


\section{Water quality parameters}

\section{Sampling location and fluoride content}

\begin{tabular}{|c|c|c|c|c|c|c|c|}
\hline $\begin{array}{c}\text { Sample } \\
\text { Number }\end{array}$ & Type & Latitude & Longitude & $\begin{array}{c}\text { Altitude } \\
(\mathrm{m})\end{array}$ & $\mathrm{pH}$ & $\begin{array}{c}\text { Fluoride } \\
(\mathrm{mg} / \mathrm{L}) \\
\text { Ionic } \\
\text { chromatography }\end{array}$ & $\begin{array}{c}\text { Fluoride } \\
\text { (mg/L) } \\
\text { Proposed } \\
\text { method }\end{array}$ \\
\hline 1 & Groundwater & -3.376808 & 36.664889 & 1377 & 7.31 & 2.8 & $2.4 \pm 0.8$ \\
\hline 2 & Groundwater & -3.407544 & 36.675525 & 1312 & 7.48 & 5.6 & $5.8 \pm 0.7$ \\
\hline 3 & Groundwater & -3.445592 & 36.706356 & 1245 & 8.01 & 7.2 & $8 \pm 1$ \\
\hline 4 & Groundwater & -3.370583 & 36.712331 & 1435 & 6.76 & 0.7 & $0.8 \pm 0.7$ \\
\hline 5 & Groundwater & -3.400758 & 36.658033 & 1328 & 7.71 & 4.1 & $3.8 \pm 0.7$ \\
\hline 6 & Groundwater & -3.346325 & 36.684417 & 1504 & 7.09 & 2.1 & $2.8 \pm 0.7$ \\
\hline 7 & Groundwater & -3.390725 & 36.736778 & 1355 & 7.33 & 1.5 & $1.3 \pm 0.6$ \\
\hline 8 & Groundwater & -3.3655 & 36.716786 & 1465 & 6.88 & 0.8 & $0.6 \pm 0.6$ \\
\hline 9 & Groundwater & -3.397025 & 36.724722 & 1323 & 7.15 & 1.6 & $1.0 \pm 0.7$ \\
\hline
\end{tabular}

Table S2a - Sampling location, $\mathrm{pH}$ and fluoride content of natural water samples.

\begin{tabular}{|c|c|c|c|c|c|c|c|}
\hline \multirow{2}{*}{$\begin{array}{c}\text { Sample } \\
\text { Number }\end{array}$} & \multicolumn{3}{|c|}{$\begin{array}{c}\text { Anions } \\
(\mathrm{mg} / \mathrm{L})\end{array}$} & \multicolumn{4}{c|}{$\begin{array}{c}\text { Cations } \\
(\mathrm{mg} / \mathrm{L})\end{array}$} \\
\cline { 2 - 8 } & $\mathrm{Cl}^{-}$ & $\mathrm{NO}_{3}{ }^{-}$ & $\mathrm{SO}_{4}^{-}$ & $\mathrm{Na}^{+}$ & $\mathrm{K}^{+}$ & $\mathrm{Mg}^{2+}$ & $\mathrm{Ca}^{2+}$ \\
\hline 1 & 67.0 & 198.9 & 46.7 & 167.6 & 67.4 & 14.3 & 56.9 \\
\hline 2 & 68.3 & 53.4 & 24.7 & 221.0 & 40.6 & 11.6 & 36.6 \\
\hline 3 & 15.7 & 10.3 & 6.4 & 178.6 & 17.0 & 2.6 & 7.4 \\
\hline 4 & 40.1 & 147.5 & 25.1 & 43.8 & 15.8 & 23.1 & 53.1 \\
\hline 5 & 17.9 & 8.1 & 17.0 & 217.3 & 29.5 & 6.8 & 18.4 \\
\hline 6 & 52.2 & 127.9 & 48.5 & 122.9 & 33.0 & 12.9 & 35.9 \\
\hline 7 & 30.8 & 50.0 & 16.3 & 31.8 & 5.2 & 22.2 & 39.8 \\
\hline 8 & 30.0 & 95.6 & 8.4 & 34.9 & 10.2 & 13.8 & 37.5 \\
\hline 9 & 27.5 & 56.3 & 3.2 & 52.4 & 10.0 & 27.4 & 49.7 \\
\hline
\end{tabular}

Table S2b-Anion and cation content of natural water samples.

\begin{tabular}{|c|c|c|c|c|}
\hline $\begin{array}{c}\text { Sample } \\
\text { Number }\end{array}$ & $\begin{array}{c}\mathrm{EC} \\
(\mathrm{mS} / \mathrm{m})\end{array}$ & $\begin{array}{c}\text { ORP } \\
(\mathrm{mV})\end{array}$ & $\begin{array}{c}\text { DO } \\
(\mathrm{mg} / \mathrm{L})\end{array}$ & $\begin{array}{c}\text { Alkalinity } \\
(\mathrm{meq} / \mathrm{L})\end{array}$ \\
\hline 1 & 136.5 & 197 & 1.72 & 6.26 \\
\hline 2 & 169.9 & 218 & 1.76 & 8.95 \\
\hline 3 & 78.4 & 175 & 3.92 & 7.25 \\
\hline 4 & 69.7 & 268 & 2.22 & 2.72 \\
\hline 5 & 105.7 & 174 & 2.13 & 9.57 \\
\hline 6 & 91.0 & 158 & 3.62 & 4.10 \\
\hline 7 & 50.6 & 184 & 2.63 & 3.04 \\
\hline 8 & 48.5 & 180 & 1.85 & 1.95 \\
\hline 9 & 66.7 & 176 & 1.56 & 5.10 \\
\hline
\end{tabular}

Table S2c - Electrical conductivity (EC), Oxidation Reduction Potential (ORP), dissolved oxygen (DO) and alkalinity of natural water samples. 
Arduino circuit

Connection on the protoboard

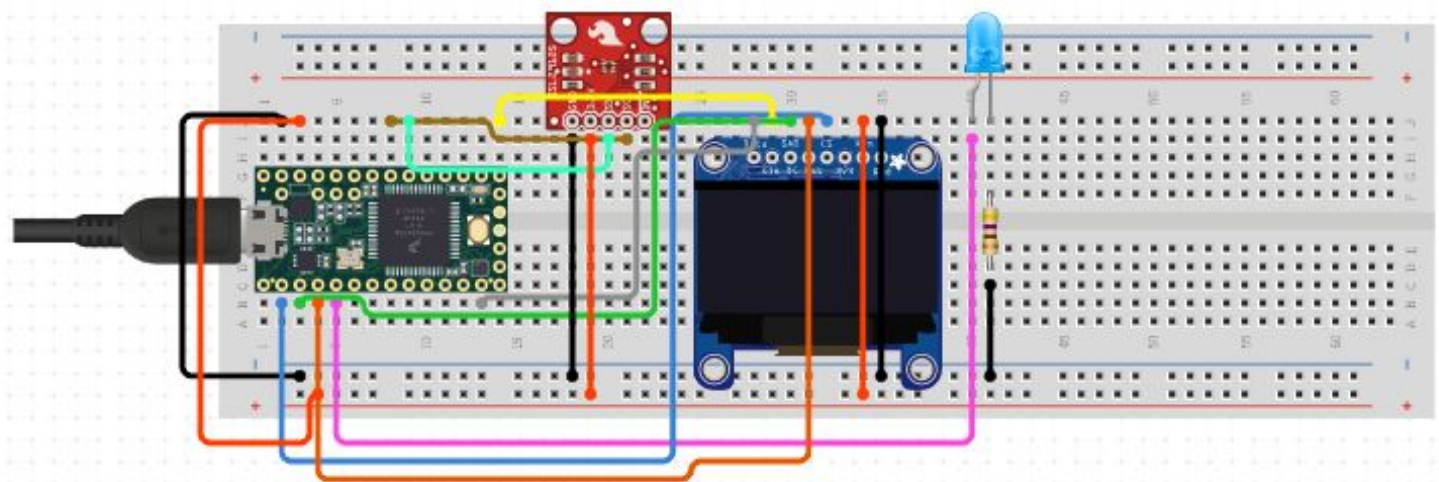

\begin{tabular}{|c|c|c|}
\hline Arduino & OLED & ISL29125 \\
\hline $5 \mathrm{~V}$ & VCC & -- \\
\hline $3 \mathrm{~V} 3$ & -- & $3.3 \mathrm{~V}$ \\
\hline GND & GND & GND \\
\hline A4 & SDA & SDA \\
\hline A5 & SCL & SCL \\
\hline
\end{tabular}

Fig S5 - Connection diagram used in the Arduino circuit

PCB design

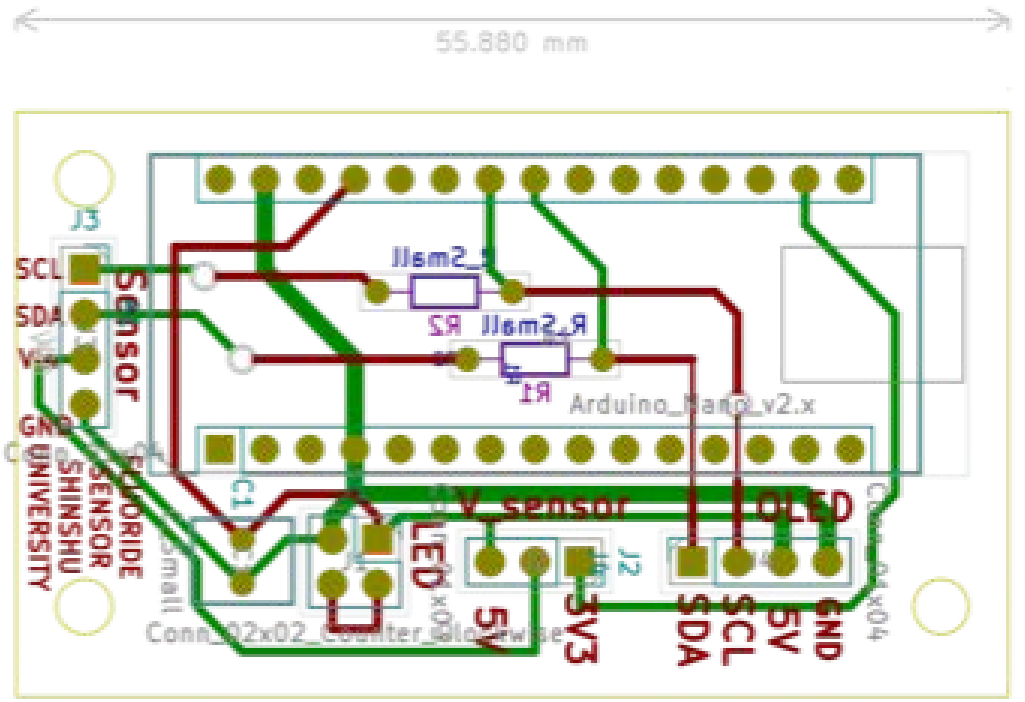

Figure S6 - PCB design for the fluoride sensor. R1 and R2 are $100 \Omega, \mathrm{C} 1$ is $0.1 \mu \mathrm{F}, \mathrm{LED}$ is a socket for the LED and a resistance to control the light emission intensity, and $\mathrm{V}$ _sensor is a jumper to regulate voltage for the sensor, ISL29125 sensor is 3.3V. 
Calibration curve using ISL29125

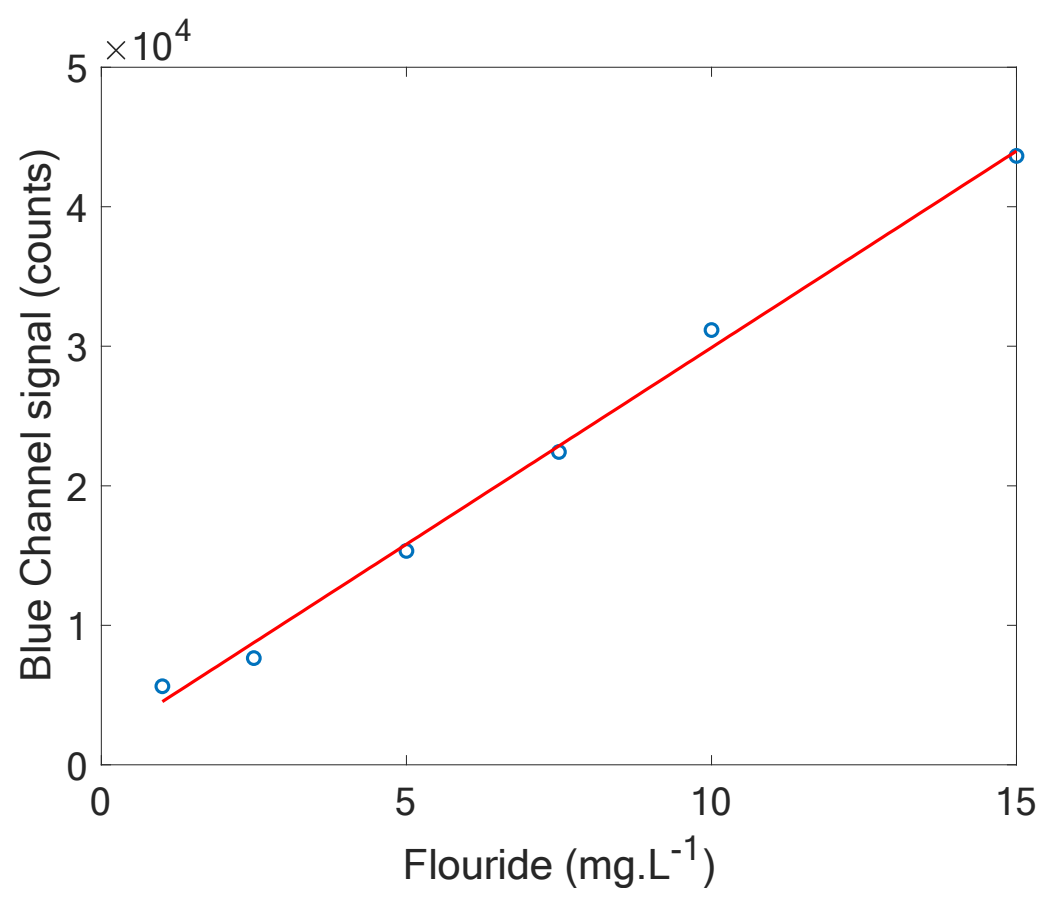

Fig S7 - Calibration curve of Fe-SCN / fluoride system in solution using ISL29125 sensor as detector and OSB5DL3E34B LED as light source.

\section{Arduino program}

Full code to use an OLED screen and serial port data export.

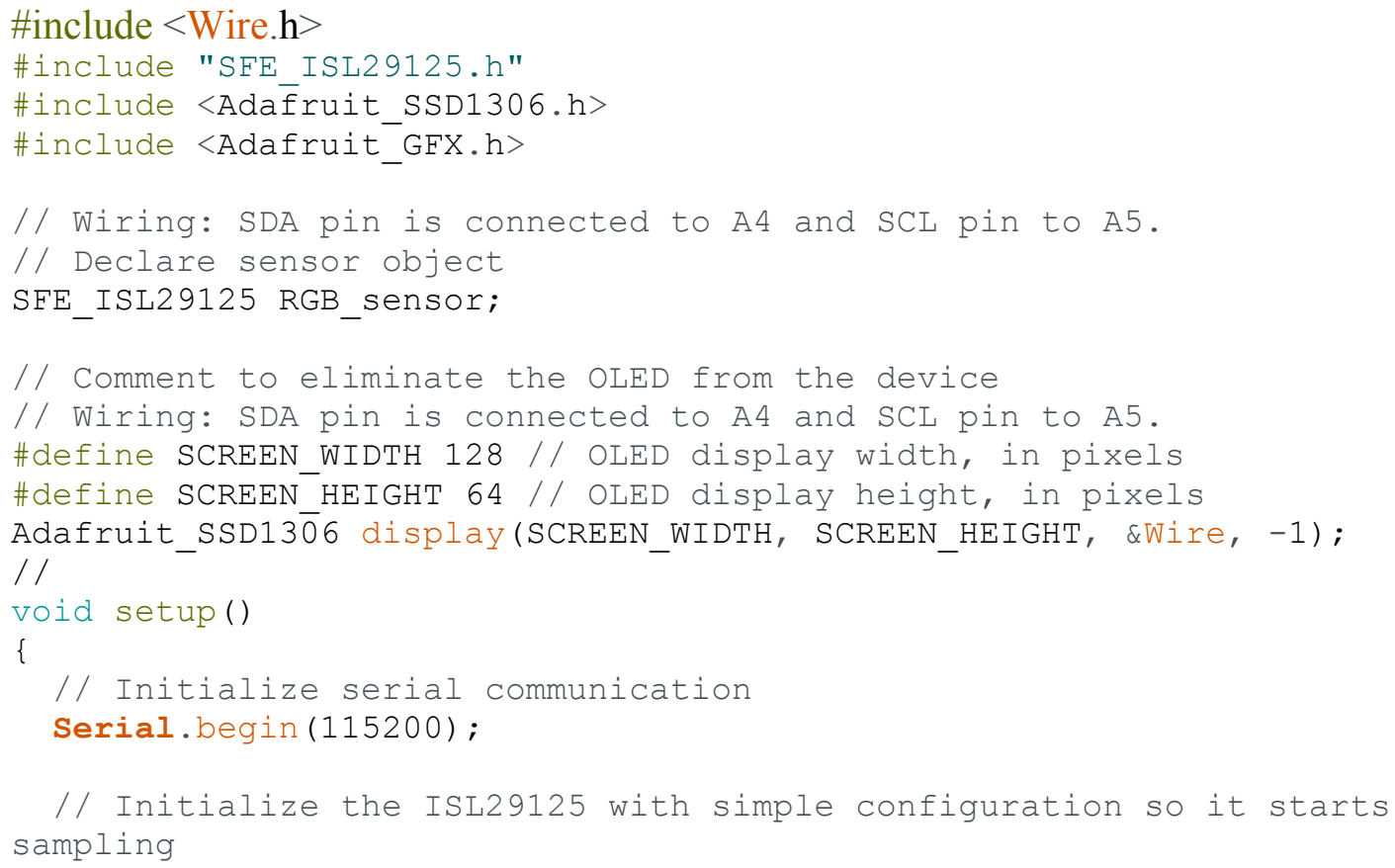




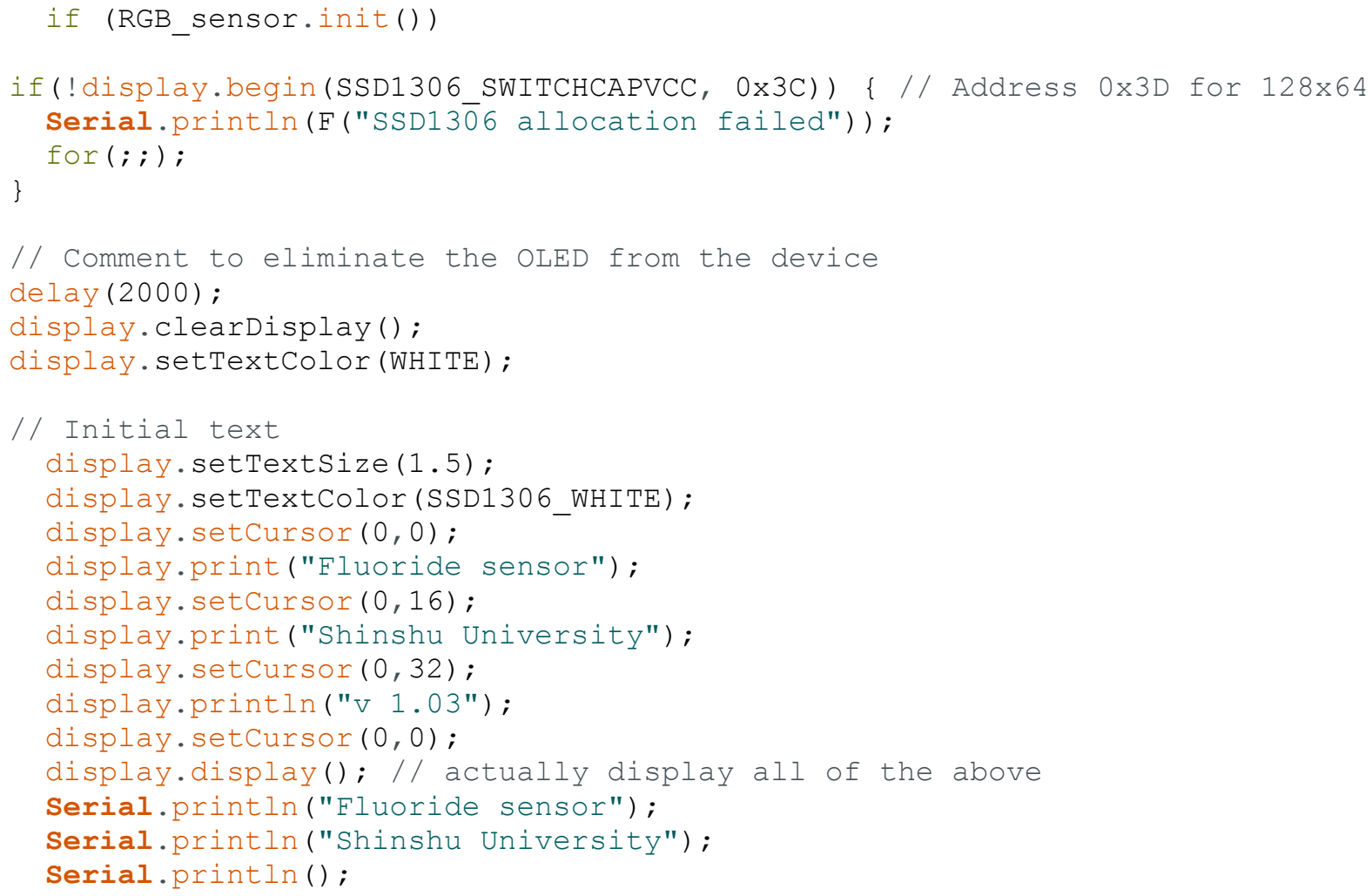




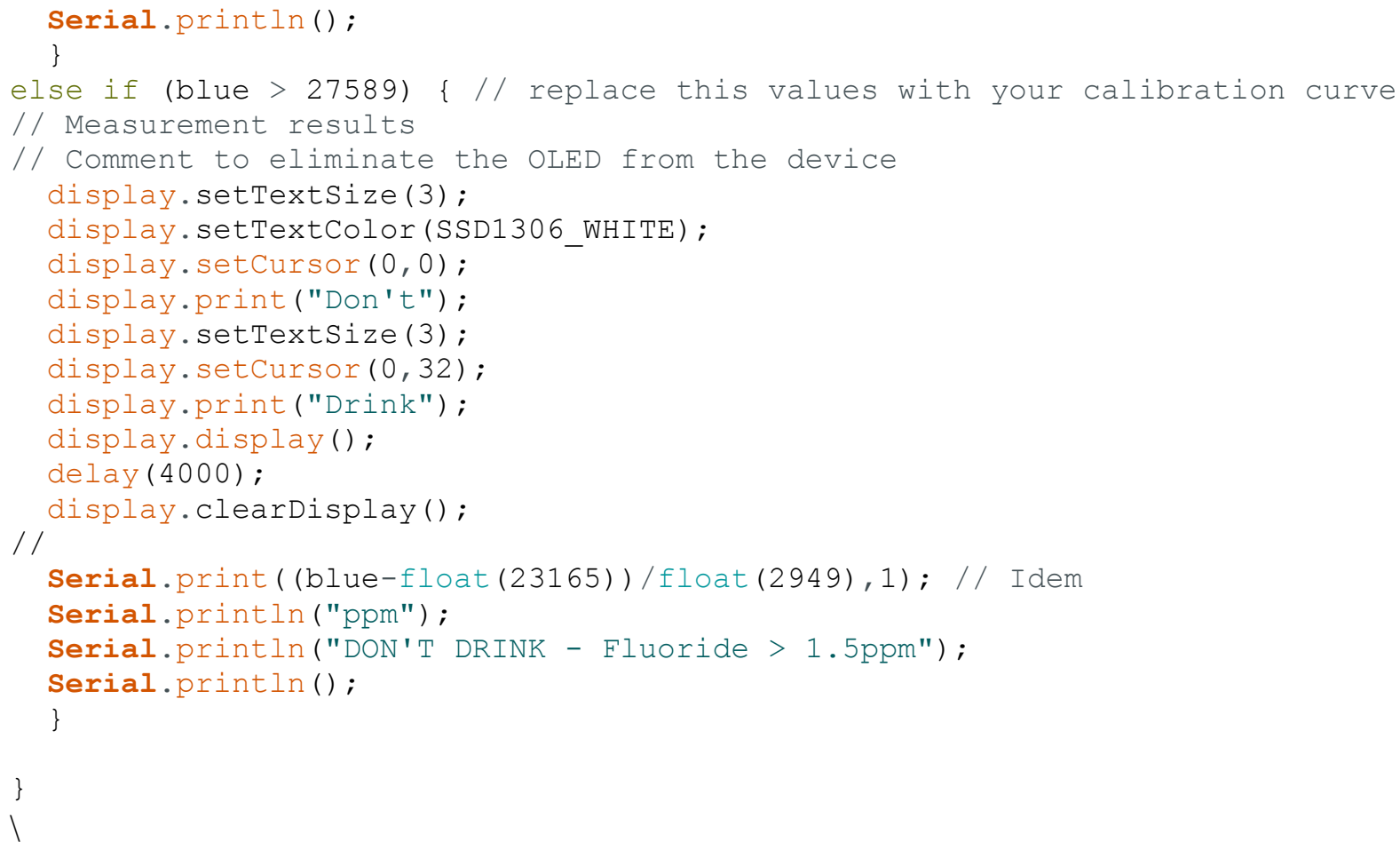

Chemical interferences

\section{Anions}

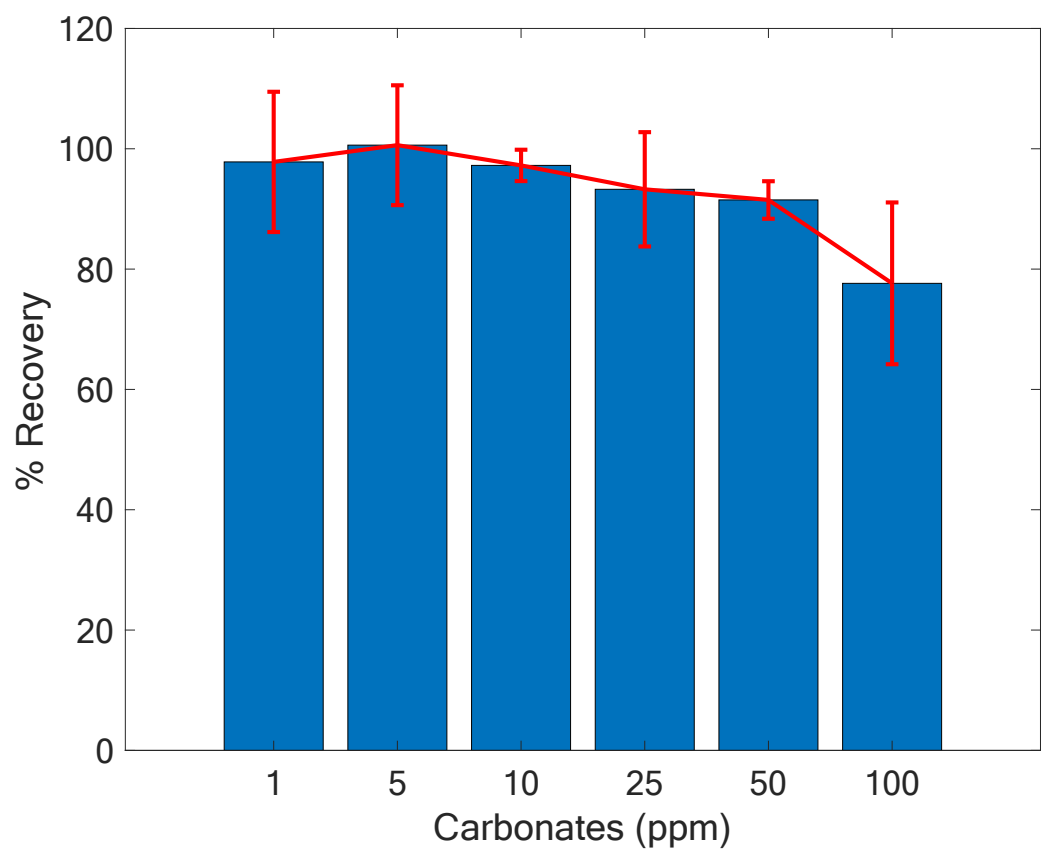

Fig S8 - Influence of carbonate interference in fluoride quantification, [F- $]=5 \mathrm{ppm}$. 


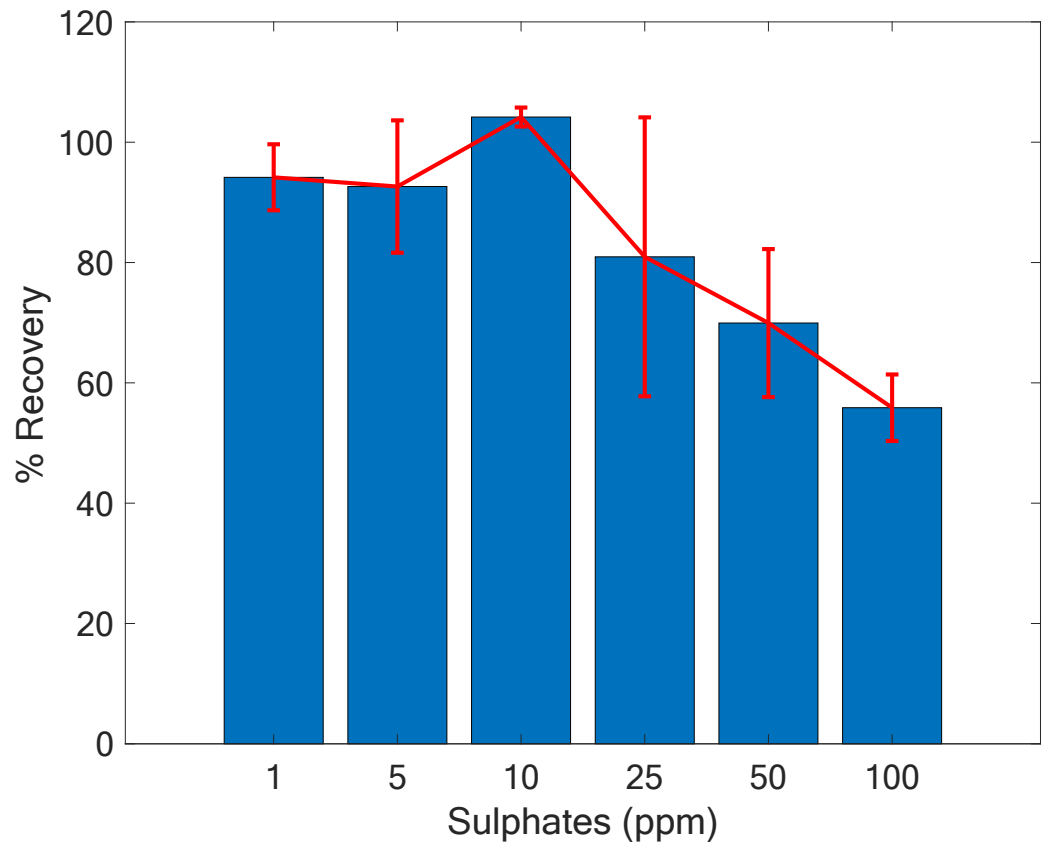

Fig S9 - Influence of sulphate interference in fluoride quantification, $\left[\mathrm{F}^{-}\right]=5 \mathrm{ppm}$.

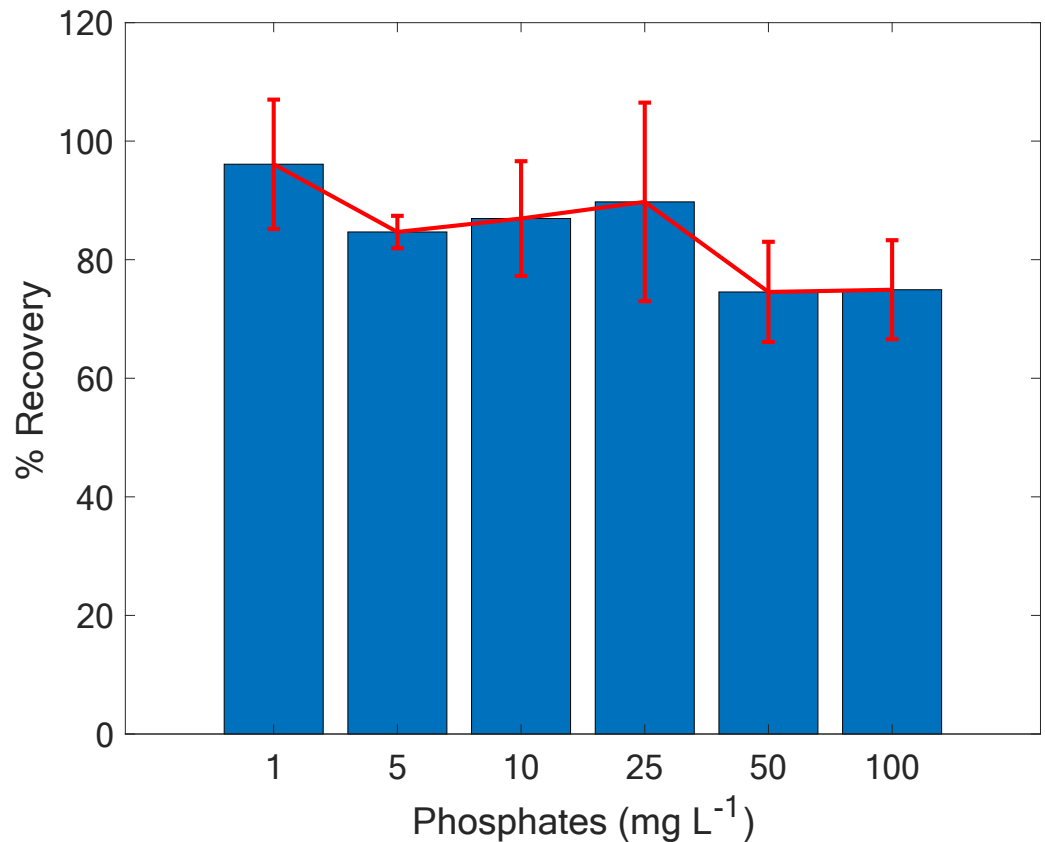

Fig S10 - Influence of phosphate interference in fluoride quantification, $\left[\mathrm{F}^{-}\right]=5 \mathrm{ppm}$. 


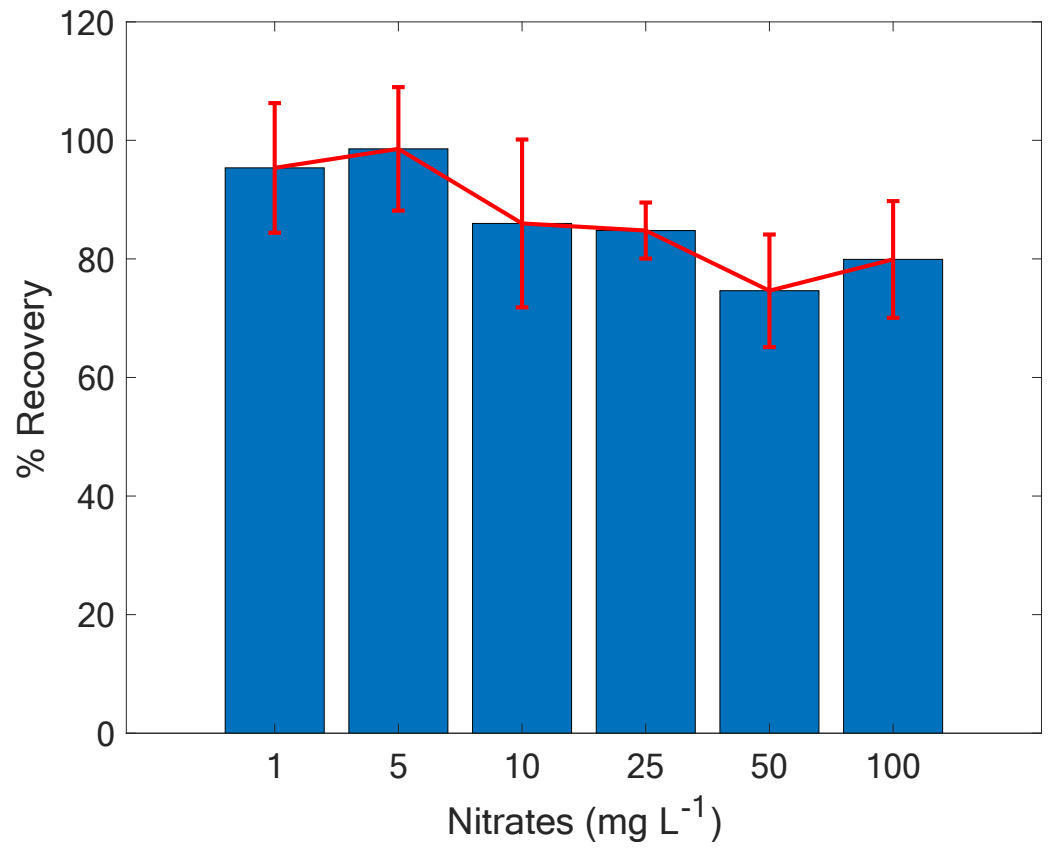

Fig S11 - Influence of nitrate interference in fluoride quantification, $\left[\mathrm{F}^{-}\right]=5 \mathrm{ppm}$.

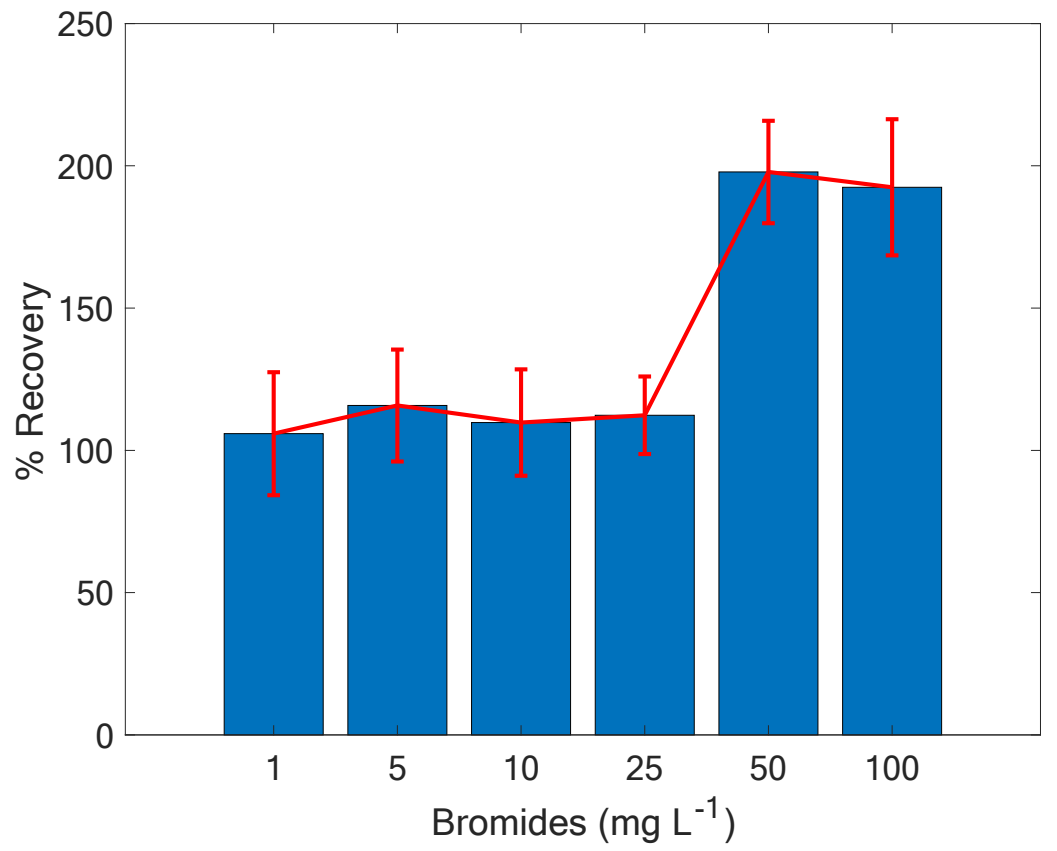

Fig S12 - Influence of bromide interference in fluoride quantification, $\left[\mathrm{F}^{-}\right]=5 \mathrm{ppm}$. 


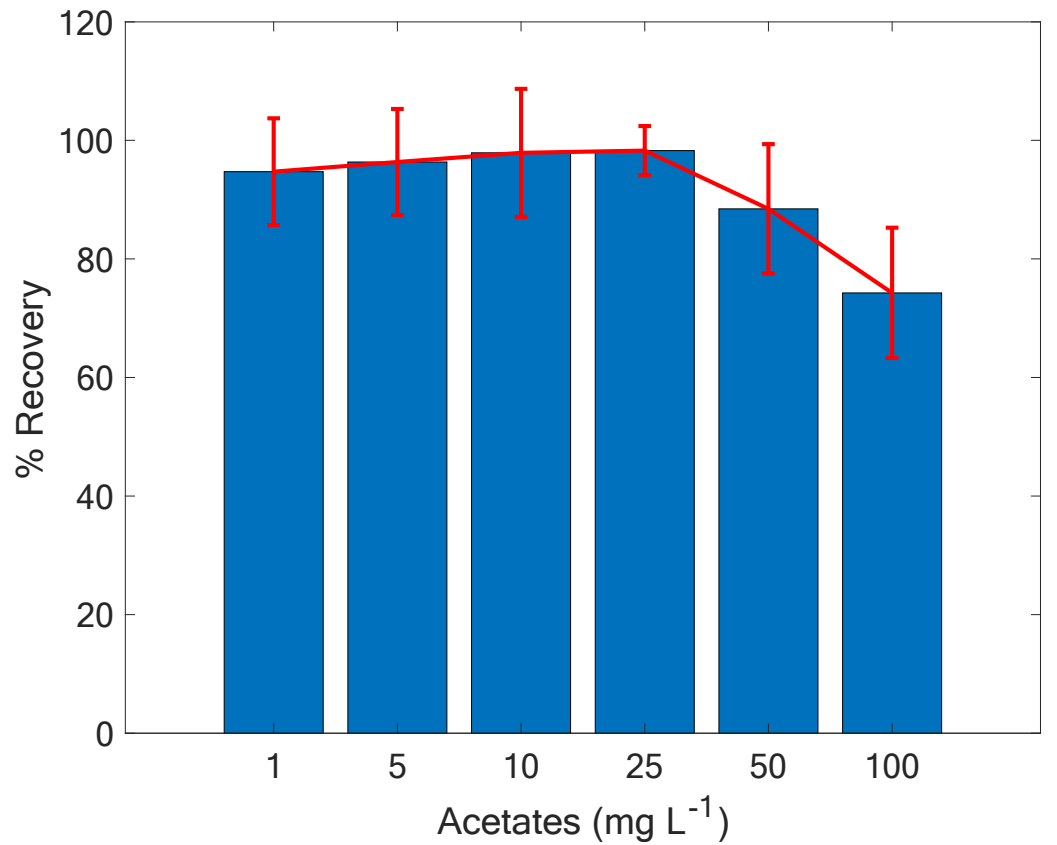

Fig S13 - Influence of acetate in fluoride quantification, $\left[\mathrm{F}^{-}\right]=5 \mathrm{ppm}$.

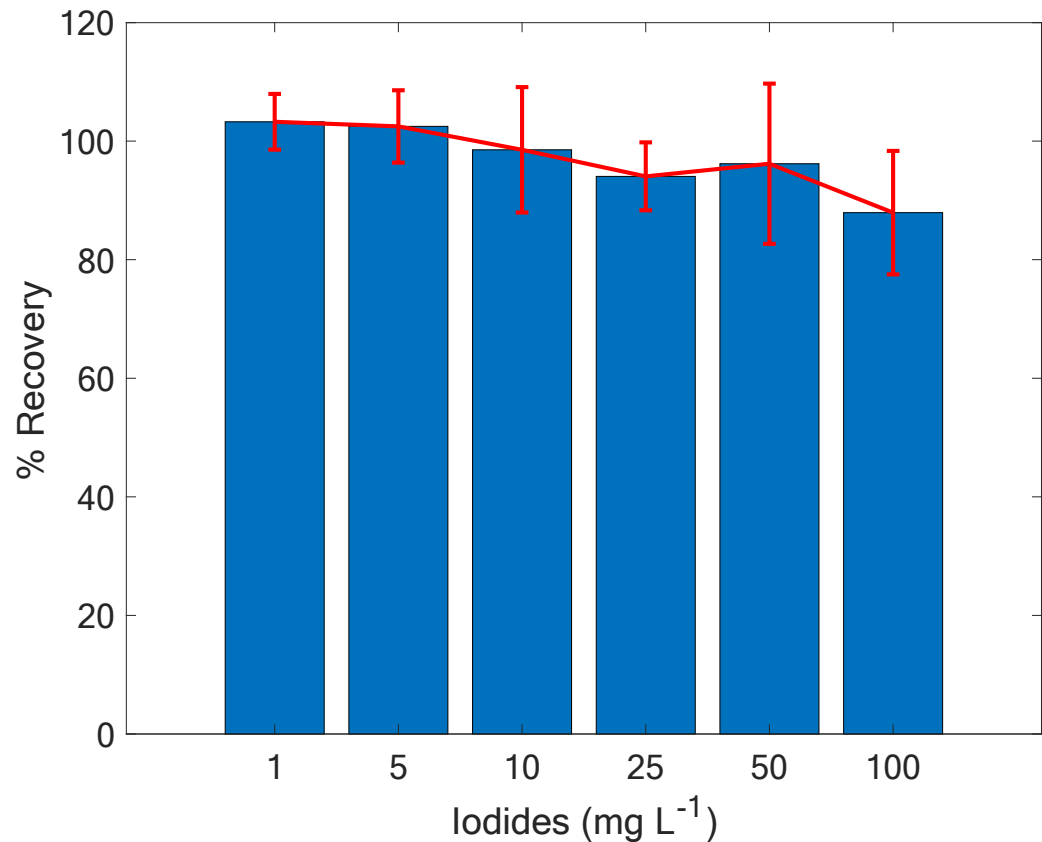

Fig S14 - Influence of iodide in fluoride quantification, $\left[\mathrm{F}^{-}\right]=5 \mathrm{ppm}$. 


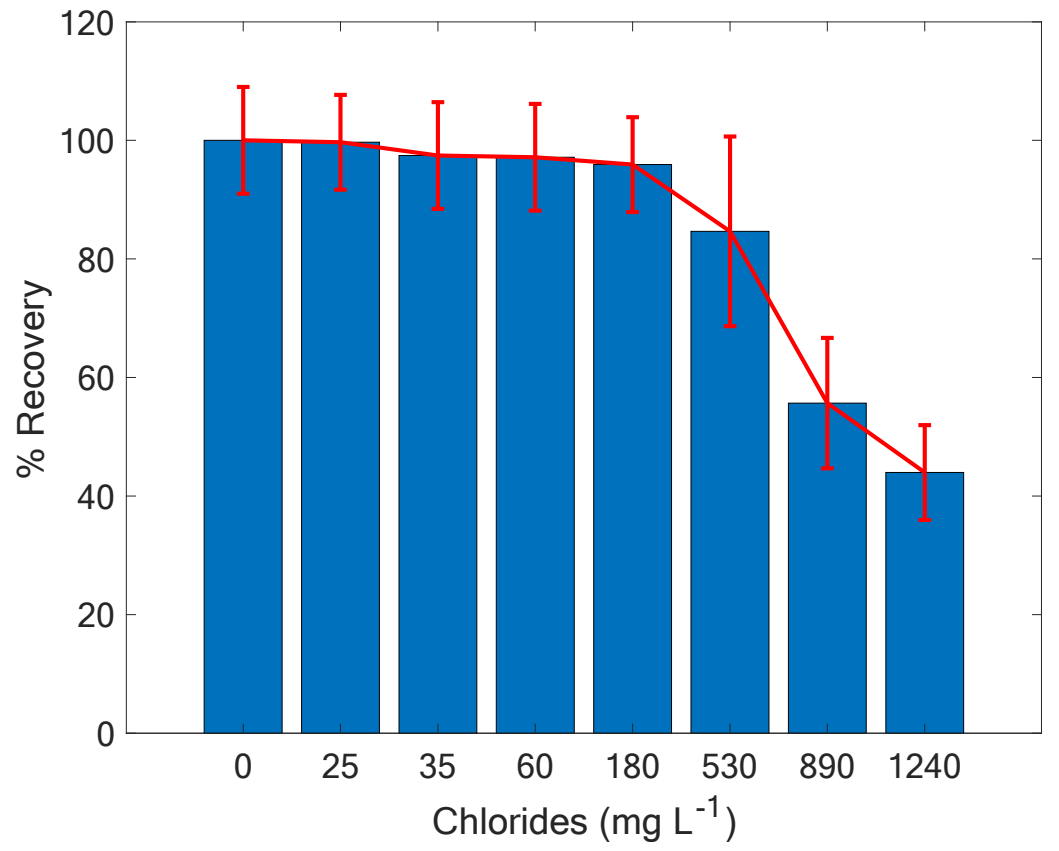

Fig. S15- Influence of Chlorides in fluoride quantification, $\left[\mathrm{F}^{-}\right]=5 \mathrm{ppm}$

Cations

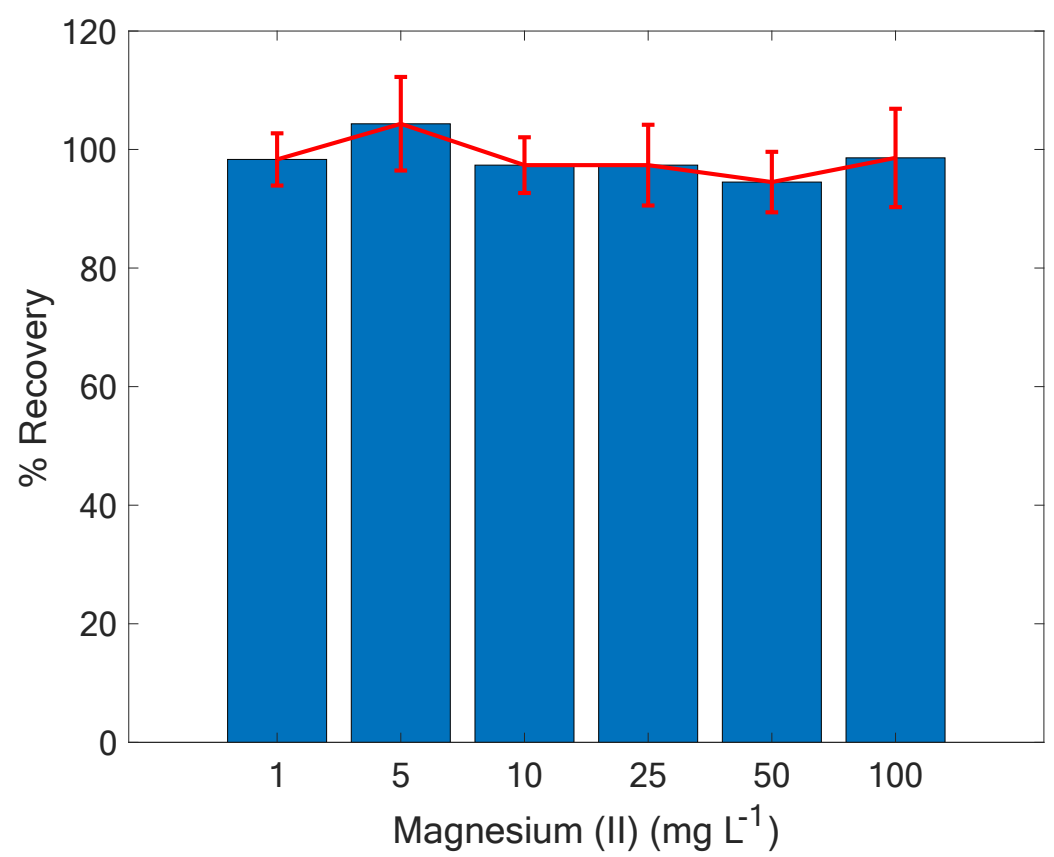

Fig S16 - Influence of magnesium (II) interference in fluoride quantification, $\left[\mathrm{F}^{-}\right]=5 \mathrm{ppm}$. 


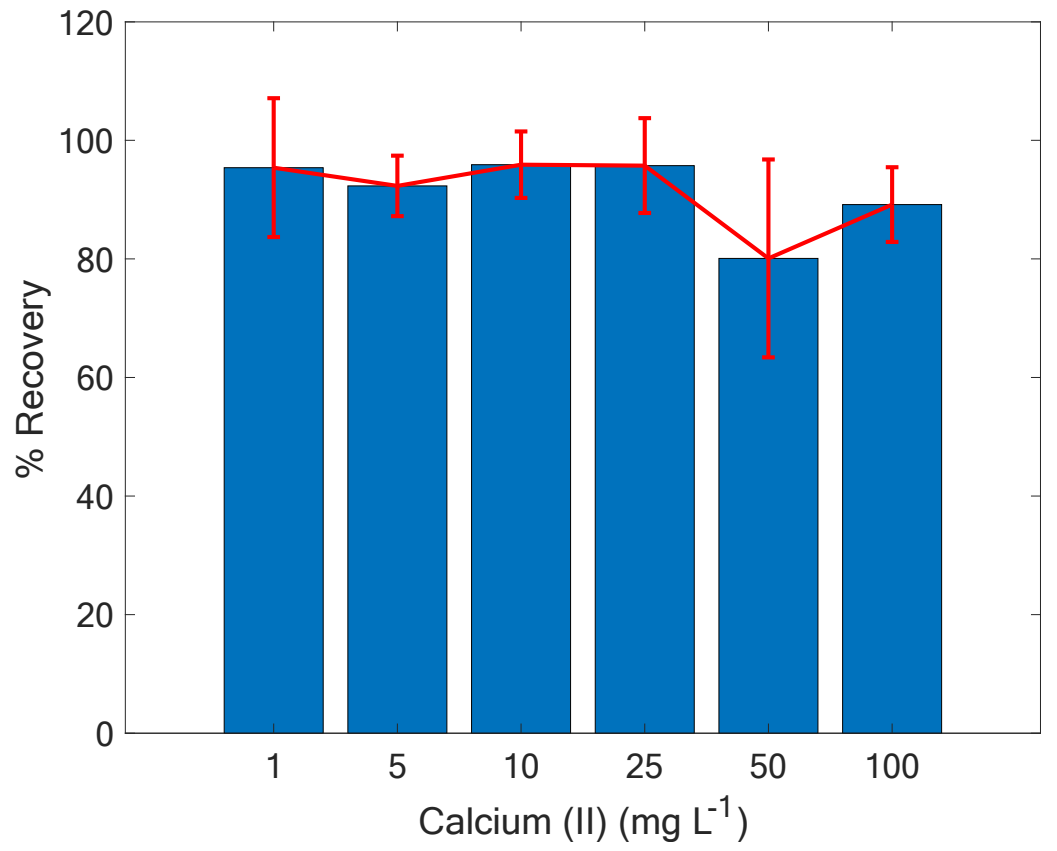

Fig S17 - Influence of calcium (II) interference in fluoride quantification, $\left[\mathrm{F}^{-}\right]=5 \mathrm{ppm}$.

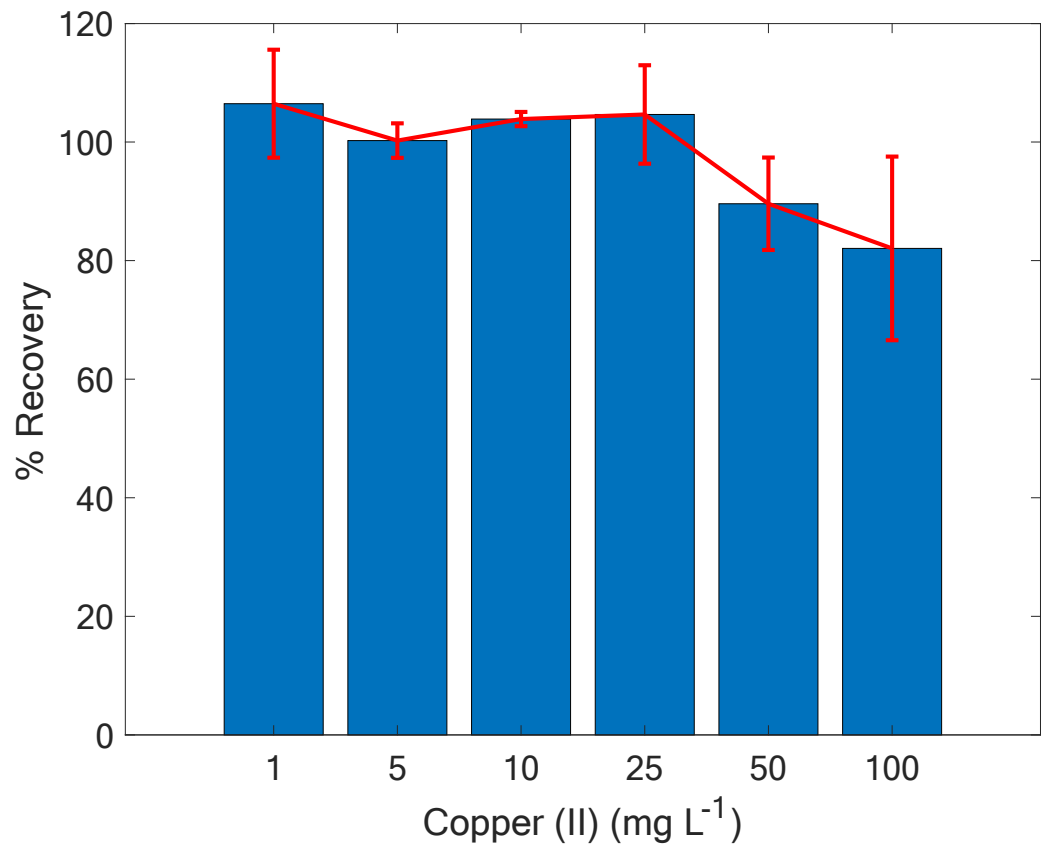

Fig S18 - Influence of copper (II) interference in fluoride quantification, $\left[\mathrm{F}^{-}\right]=5 \mathrm{ppm}$. 


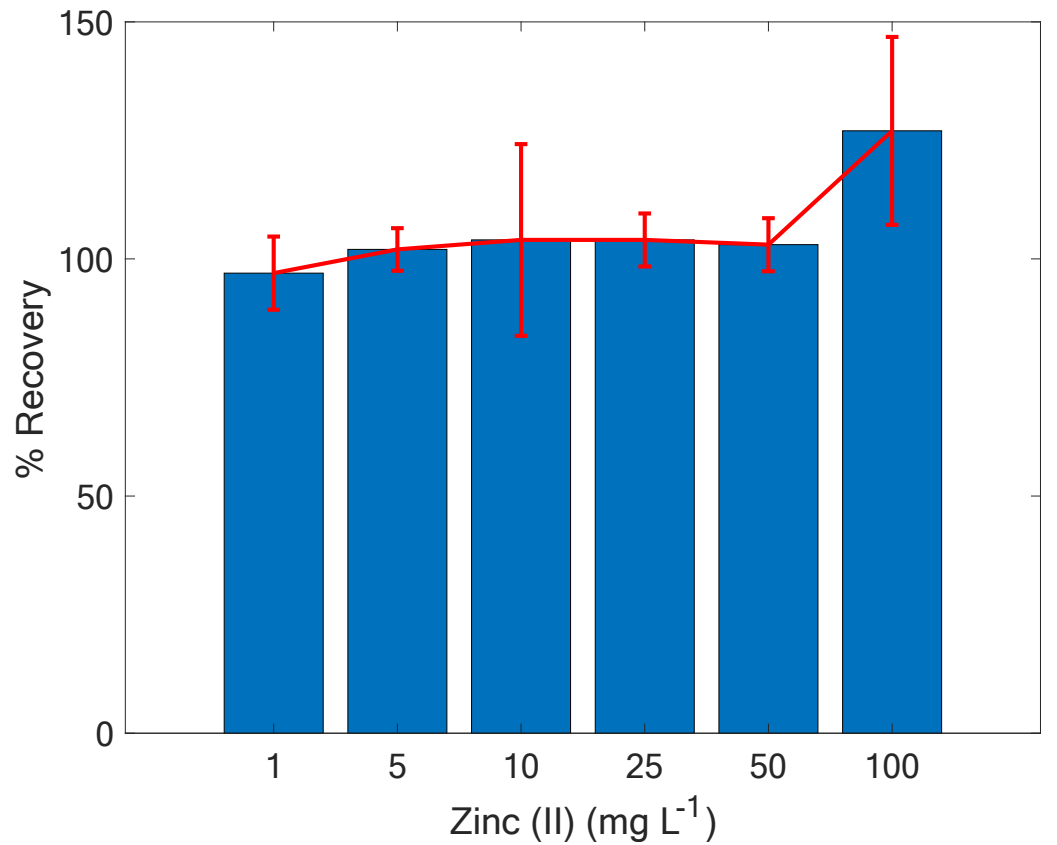

Fig S19 - Influence of zinc (II) interference in fluoride quantification, $\left[\mathrm{F}^{-}\right]=5 \mathrm{ppm}$.

\section{References}

(1) Complexation of Iron(III) with Thiocyanate lons in Aqueous Solution https://www.jstage.jst.go.jp/article/analsci1985/8/4/8_4_521/_article (accessed Jun 8, 2020).

(2) Arribas Jimeno, S.; Hernandez Mendez, J.; Lucena Conde, F.; Burriel Marti, F. Química analítica cualitativa, Paraninfo.; 2002. 